\title{
XXVII. On the barometer
}

\section{Richard Walker Esq.}

To cite this article: Richard Walker Esq. (1812) XXVII. On the barometer, Philosophical Magazine Series 1, 40:172, 136-144, DOI: 10.1080/14786441208638203

To link to this article: http://dx.doi.org/10.1080/14786441208638203

$$
\text { 曲 Published online: } 27 \text { Jul } 2009 .
$$

Submit your article to this journal 전

LII Article views: 2

Q View related articles $\asymp$ 
that used to saturate an equal weight of the same acid diluted with water, and I find them to agree exactly.

M. Vogel*, in his remarks on this suhject, seems to think that increasing the quantity of acid bastened the formation of the sugar. He only obtained the syrup; time did not permit him to go further: we are therefore, for the present, deprived of that able chemist's remarks on this subject. Should my future experiments on this subject prove sufficiently interestiug, I shall have great pleasure in making them public, and I will take the liberty of asking indulgence for these, as I am but a pupil in that science in which there are at present so many able proficients. If these remarks should induce some able investigator to pursue the subject, and thereby benefit science and the arts, I shall consider myself as very fortunate.

XXVII. On the Barometer. By Richard WALKER, Esq. Oxford. Communicaled by the Author!

$$
\text { To Mr. Tilloch. }
$$

Sir, Syould the following observations, forming the conclusion of a subject, viz. "Observations on the Barometer," which you did me the favour to communicate, through the channel of the Philosophical Magazine for October 1810, and the two following numbers, be deemed not unw orthy of a place in your next number, I request you will have the goodness to insert them.

Oxford, August 17, 1812 . I am, sir, your obedient servant, RichaRd Walker.

P. S.-I have judged it expedient, in the course of these observations, to descend occasionally to particulars which, to the generality of your readers, must be totally unnecessary, but which to some persons, who may not before have given much attention to this subject, may not prove unacceptable.

The reason of the long interval between this and my former communications on the same subject, is in consequence of my attention having been engaged in a small work of a different nature, which 1 hope soon to have it in my power to announce.

The barometer, stationary, with a concave surface of the mercury at the top, is an almost infallible indication of

- Journal de Physique Mars 1812, Notice sur la tranemutation de fecula de frument en matière sucrée, par $M$. Vogel. 
rain, at the place of observation, or in its vicinity ; especially if the barometer be at or below CHANGEABLE.

The barometer, stationary, with a convex surface of the mercury at the top, is a strong indication of fair weather, at the place of observation, or in its vicinity; especially if the barometer be at or alove CHANGRABLE.

In the first instance, I would account for this circumstance, by the natural spring or elasticity of the air being suspended or diminished (the density or weight of the atmosphere remaining the same) by the intervention of vapour collecting into a mass; and in the latter instance, from the dispersion of the interposed vapour, by which the natural spring or elasticity of the air is restored.

For the same reason it is, I presume, that during a steady fall of rain, the barometer is commonly stationary, with a concave surface at the top; and as soon as the weather begins to clear up, the top of the mercury in the barometer assumes a convex surface.

The quick ascent into the atmosphere, of smoke, vapour, \&cc. indicates fair-weather; and the slow ascent, and particularly the descent of them, indicates rain. The former, in consequence of an influx of denser or heavier air, into the lower stratum of the atmosphere, commonly from the north and east points ; and the latier, in consequence of an influx of rarer or lighter air, into the lower stratum of the atmosphere, commonly from the south and west points.

Upon the whole there is more wet weather, whilst the barometer is alove CHANGEABLE; than there is dry weather, whilst the barometer is lielow changeable. Hence it follows that, cateris parilus, the former state of the barometer is not so strong an indication of fair weather, as the latter is of rainy weather.

The barometer rising, especially if it be progressive, whilst the wind is in the south or south-west points, indicates a change of the wind to the north or east points; and conversely, the barometer sinking, whilst the wind is in the opposite points, indicates a change to the south or west points.

In the spring, and as summer advances, rain brings successively warmer weather after it; and in autumn, and as winter approaches, rain brings successively colder w'ealher after it. Hence, successive showers gradually change, as it were, spring into summer, and autumn into winter.

The reason of this seems to be, that during fair weather alone, the true character of each season, with respect to temperature, 
temperature, is exhibited; whereas it is in some degree obscured, or suspended, during cloudy weather.

The mercury in the barometer is usually fixed, at whatever height it may happen to be, with a concave surface, during settled rain; and fixed with a convex surface, during setiled fair weather. It is essential, in order to make accurate observations on the barometer, that it be viewed with a magnifier, which is best when fixed to the moveable index.

Fogs in the morning are more visible over land than over water, beeause the greater colduess of the land condenses the vapour most; but in the crening, over the water, because water is then the coldest.

The mean height of the clouds above the carth's surface is about three quarters of a mile; - the greatest height, somewhat more than a mile-and the least height, ahout three furlongs, and sometimes in thunder storms, even less.

In order to make the most accurate observations on the state of the weather, and to predict the various changes which are about to take place in it, the observer should be provided with a barometer, thermometer, and hygrometer, each of the best construction. De Luc's hygrometer, I think, is the best; - and in order, at all times, to be able to notice the changes in the direction of the wind, which are taking place, and which is extremely essential, it would be expedient that a wind-vane be so constructed, as to exhibit the direction of the wind within the room appropriated for meteorological observations; which, in defect of any better means, might be contrived, by the axis of the wind-vane, without being continued so as to reach within the room, having an index affixed to it.

The rising of the barometer is a more certain indication of fair weather, than its sinking is of rainy weather; because it sinks for wind, as well as rain. If therefore, whilst the barometer is sinking, the atmosphere still remain clear, wind may be expected.

In winter, spring, and autumn, a sudden falling of the mercury presages high winds and storms; but in summer, heavy showers, and sometimes thunder.

The principal changes in the atmosphere take place about the time of the vernal equinox, viz. from the 16 th to the 28th of March.

Rain or snow may come during a north and easterly wind, whilst the barometer stands higher, than when the wind is in the south or uest points; because in the former instance 
instance the clouds come from a denser to a rarer medium, and in the latter case, from a rarer into a denser medium.

The maximum and minimum heights of the barometer, in this and every other country, are limited, by nature, thus : When the atmosphere is charged with as much water in a state of chemical combination, under the most favourabie circumstances for absorbing it, as the atmosphere can sustain, it is then heaviest, and the barometer is at its maximum;-and when the air is most free from, or contains the least quantity of, water in a state of chemical combination, other circumstances concurring, the atmosphere is then lightest, and the barometer is at its minimum of height. Hence, cateris pariluts, the absorption of water, by air, renders the atmusphere heavier, and its parting with it renders it lighter*.

During many successive years of observation on the barometer in England, it may be found, that its greatest height will not exceed one inch and a quarter above CHANGEABLE; nor its least height, the same distance below. CHANGEABLE.

The variations in the barometer are greatest towards the poles; less, in temperate latitudes; and least, between the tropics.

The barometer was observed, some years ago, at Oxford, as low as 28 inches, within two hundredths, at which time the weather was perfectly fair and calm; but it appeared afterwards that an earthquake had happened at the time, at a distant part of the world. This circumstance puzzled ordinary observers, and injured the reputation of the weather-glass; but the intelligence afterwards of the event which had happened restored its character.

The moisture on the ground and in other situations freezes, as is well known, when the atmosphere is warmer than $32^{\circ}$, the freezing point, in consequence of the cold produced by evaporation. Under the most favourable circumstances, viz. a dry air and a lrisk wind, this may happen, when the air is eight or ten degrees above the freezing point. The rise or fall of one-tenth of an inch, in the barometer, is usually accompanied, or followed, by a very perceptible change in the weather.

* Among other circumstances, which show the power of air for absorbing water or vapour, the following is a very curious as well as satisfactory nne : if a small cloud, or fragment of a cloud, in a loose or rare state, insulated from others, in a clear part of the atmosphere, especially when the air is warm and calm, be observed, it will be found to melt, or dissolve, as it were, in the atmosphere, gradually, and at length to vanish entirely. 
Rain happenc, among otber causes before mentioned, in consequence of a subtraction of the electric fluid, which, when the air abounds with vapour, is easily conducted to the earth; hence arises the velocity of rain in thunder showers.

In this climate, the hottest time of the day is about three in the afternoon, in consequence of the continued influence of the sun, whilst near its greatest height from the horizon; and the coldest is a short time lefore sun-rise,viz. that time at which our part of the earth has been longest without the influence of the sun.

The hottest month is July; and the coldest month is January; the former, in consequence of the continued strongest influence of the sun, whilst near its greatest elevation from the horizon; and the latter, in consequence of the continued weakest influence of the sun, whilst near its least elevation from the horizon. The driest months are July and August, and the wettest are Octoler and November; the former, in consequence of the small quantity of water which has been raised into the atmosphere previously, and the powerful influence of the sun, during these months, in dissipating the vapours which are raised; and the latter, in consequence of the inverse effects of those two causes.

In like manner, as the annual influence of the sun operates upon the weather, producing different seasons, respecting wet and dry weather, before stated; it is not improbable, that its diurnal influence, being a kind of epitcme of the former, may, in a smaller degree, have the same effect in producing an effort for fair weather, whilst the sun is nearest its greatest diurnal altitude, in consequence of its greater power, at that time, of dissipating vapours, or dispersing clouds.

The greater or less avidity with which the mercury appears to rise or sink, as exhibited by the greater or less convexity or concavity of the mercury at the time of observation, the stronger or weaker will be the indication in each instance.

The surface of the mercury in a barometer is best seen when the tube, at, the part to be observed, is exposed to the light behind, that is, constructed without an opake substance at the back of it.

The convex or concave surface of the mercury arises, of course, from the adhesion of the mercury to the tube, in rising or sinking: at each observation of the barometer, therefore, after noticing the variation in height, the index should 
should be first accurately placed, the barometer then smartly ripped, in order to reduce the mercury to a level, and the index again accurately adjusted.

The principle of a barometer is so perfectly. uniform in its effect, that although, for want of precision in their construction, any two of them may not, under similar circumstances, respecting the state of the atmosphere, and the elevation in which they are placed, stand precisely at the same height; $y \in t$ all will agree in this-they will rise or sink at the same time.

The ordinary upright barometer is the only one in use, among scientific persons, of which there are two kinds, viz. the cistern or bason barometer, and the syphon barometer: the former of these requires a floating gage, with an adjusting screw, in order to show the precise length or beight of the column of mercury in the tube; which is essential in accurate observations, and particularly in the measurement of altitudes by the barometer; but which may be dispensed with in ordinary observations.

The only objectionable circumstance in the perpendicular larometer is the shortness of its scale; but, since there are no means of extending the scale of a barometer without, in some degree, injuring its principle, this defect is supplied by a nonius or vernier.

Barometers intended for the purpose of measuring altitudes, are fitted up in a peculiar manner adapted to that intention.

The attractive power of the sun upon the earth's surface, is to that of the moon, as 2 to 5 .

With respect to the direction of the wind, it is tolerably uniform within the tropics, being more immediately under the influence of the two principal causes of currents of air, viz. first the sun, which, in its course heating the places it successively passes over, causes a very considerable rarefaction of the air there, which is constantly followed by a current of air, to restore the equilibrium;--and secondly, the current of wind, which is produced by the attractive intluence of the sun and moon on the atnosphere, as the different places pass under them.

Near the poles, the wind is probably more uniform than it is in the middle or temperate climates; the lower portions of the air being constantly inclined towards the equator, in consequence of the increasing heat in that direction; and the upper portions being renewed, from the more elevated regions of the inter-tropical currents.

Whereas, in the middle or temperate climates, besides ather causes unknown to us, the direction of the wind be- 
comes more camplicated, in consequence of its situation between the two former; being occasionally influenced by each of them, separatelv or conjointly.

There seems to be a kind of habit in the weather, as in other cases: thus, towards the close of a series of dry weather, several efforts are apparently made towards a series of rainy weather, before it actually sets in; and inversely.

With respect to the difference of the weather, which I have presumed lakes place during the increase and wane of the moon; I would account for it on a supposition that at each conjunction of the moon with the suu, or the time of the new moon, as it is called, there may be a kind of disposition, as it were, for clarifying the atmosphere, or renovation of fair weather; and vice versâ, at the moon's upposition, or full moon; and the reason why this change does not actually take place until about the third or fourth day after each change of the moon, because, as I have stated before, the former habit of the weather is not till then entirely conquered.

The atmosphere becoming drier, as indicated by the $h y-$ grometer, indicates a disposition for fair weather, and vice versâ.

A cold, dry, and serene state of the atmosphere is most favourable for barometrical measurements.

The limit, or boundary of the atmosphere, terminates where the expansive force or power of the air, upwards, or from the earth's surface, is completely overcome by the force or power of gravitation downwards, or towards the earth's surface, which is estimated at about 44 or 45 miles*.

A pint measure of air, when the barometer is at $29 \frac{1}{2}$ in ENGLAND, weighs eight grains, or a gallon 64 grains; but near the POLES somewhat more; and at THE EQUATOR somewhat less. This variation in the weight of the air, in the different parts of the world, is ascribed to a difference in the height of the atmosphere; by which it happens, that the air near the surface of the earth, at different places, differs in density; the pressure or weight of the incumbent atmosphere being the same. The atmosphere is presumed to extend highest at the equator, and to be lowest at the poles; in consequence of a greater degree of centrifugal force and heat at the equator.

The barometer, cateris paribus, stands higher in winter than in summer, in consequence of the greater condensation of the air, from cold, at that season.

* The atmosphere, it is computed, would extend only to about $5 \frac{5}{2}$ miles in height, if it were so compressed, that its density throughout should equal its degree of density near the surface of the earth. 
The barometer unquestionably has been observed, in England, to rise alove 31 , and to sink below 28 ; viz. to exceed those limits, each way, by the tenth of an inch.

The lowest point at which I have observed the barometer is $28 \frac{x}{4}$ inches, viz. on November 9th, 1810; which was accompanied by a remarkably high wind, and torrent of rain.

The ordinary range, or variation, in the barometer, differs according to the latitude of the place:-thus, at the equator (except during the burricane season) it is scarcely any thing;-and ai the poles, it is probably not less than four inches; varying in an increasing ratio, from the equator, to each pole*.

The column of mercury in the tube of a barometer, as most persons know, is sustained by the counter-pressure of a column of air, of the same base, extending to the top of the atmosphere; which, when at its mean state of gravity, that is, when the barometer is at $29 \frac{1}{2}$ inches, presses on the base of the mercury, with a weight which is equal to, or after the rate of, fifteen pounds upon each square inch of surface.

In a comparison of barometers placed in different situations, with respect to elevation; an allowance must he made, at a mean rate, of loodth of an inch on the barometer for each complement of nine feet measure in height; that quantity being added to the higher barometer, or subtracted from the lower one, in order to make the comparison equal t. (See Table 1. Ec. page 279, of Philosophical Magazine, for Octoler 1810.)

It has been confidently asserted by respectable authors, that the barometer is affected by the atmospherical tides; it does not appear, however, that any observations have actually proved the fact.

With respect to the periodical rise and fall of the barometer noticed at Calcutta; in the first place, the variation appears to have been very small; and secondly, it does not correspond in point of time with the attractive infuence either of the sun or moon.

That the atmosphere is influenced by the attractive power of the sun and moon, there can be no question; but it seems

* The barometer at PERv, under the equator, varies ordinarily, only to the extent of two or three-tenths of an inch; but at Peterssurge, to the extent of three inches.

+ The difference in the height of two barometers equally well constructed, one being in the ground floor, and the other in the attic, will of course amount to several hundredths of an inch. 
contrary to reason, that the barometer should be affected by it; since, in proportion as the atmosphere is rarefied, from this cause; in the same proportion, from the same cause, likewise, is the atmosphere accumulated there; and hence the gravity, or pressure of the atmosphere upon the surface of the earth, remains the same in each instance.

I might add, that if an alteration in the barometer actually took place from such a cause, it could scarcely have escaped my notice.

P.S. I have noticed with considerable sensations of surprise and satisfaction, the progress of Professor Leslie, in his experiments on the production of cold by means of the airpump, in which it is stated that that gentleman has already reached a cold of $70^{\circ}$ below 0 . At the time I was pursuing my experiments on artificial cold by frigorific mixtures, the means of producing artificial cold by means of the air-pump naturally presented itself, and to which I was more particularly stimulated by an experiment related by Mr. Nairne, in which it is said, by the evaporation of ether, under the air-pump, a cold of $45^{\circ}$ below 0 was produced, the temperature of the air in the room being 55\%. Philosophical Transactions 1777 .

I made several attempts at producing an extraordinary degree of cold by this means, but without success; owing chiefly, as I presumed, to the imperfection of the best airpump I was able to procure. Conceiving that the Torricellian vacuum, as it is called, might be the best method of effecting the most sudden and complete evaporation of ether, upon which the success of the experiment depends, I had projected in my mind to put this method to the test; but the difficulty of obtaining a fit apparatus for the purpose prevented me.

Perceiving that Professor Leslie's experiments, in which he has succeeded to a most extraordinary degree, have been conducted upon the principle alone of rarefying the air, without any auxiliary means excepting his ingenious method of absorbing the vapour, the existence of which necessarily diminishes the success of the experiment; 1 have been induced to take this opportunity of mentioning these. circumstances, fully trusting that, should these observations furnish no hint to the learned and ingenious Professor, they will not be deemed an improper interference*.

- By means of evaporation in the open air, from purified sulphuric ether, (as mentioned in my Treatise on the Production of Culd, pages 80, 81, l sunk the thermometer from $71^{0}$, the temperature of the air at the time, to $12^{\circ}$; and by water used in a similar manner, to $56^{\circ}$. 